\title{
Androgen and Estrogen Receptor Imaging in Metastatic Breast Cancer Patients as a Surrogate for Tissue Biopsies
}

\author{
Clasina M. Venema*1, Lemonitsa H. Mammatas*2, Carolina P. Schröder ${ }^{1}$, Michel van Kruchten ${ }^{1}$, Giulia Apollonio ${ }^{1}$, \\ Andor W.J.M. Glaudemans ${ }^{3}$, Alfons H.H. Bongaerts ${ }^{\dagger 3}$, Otto S. Hoekstra ${ }^{4}$, Henk M.W. Verheul ${ }^{4}$, Epi Boven ${ }^{2}$, Bert van der Vegt ${ }^{5}$, \\ Erik F.J. de Vries ${ }^{3}$, Elisabeth G.E. de Vries ${ }^{1}$, Ronald Boellaard ${ }^{3}$, Catharina W. Menke van der Houven van Oordt ${ }^{2}$, \\ and Geke A.P. Hospers ${ }^{1}$ \\ ${ }^{I}$ Department of Medical Oncology, University of Groningen, University Medical Center Groningen, Groningen, The Netherlands; \\ ${ }^{2}$ Department of Medical Oncology, VU University Medical Center, VUmc Cancer Center Amsterdam, Amsterdam, The Netherlands; \\ ${ }^{3}$ Department of Nuclear Medicine and Molecular Imaging, University of Groningen, University Medical Center Groningen, \\ Groningen, The Netherlands; ${ }^{4}$ Department of Radiology and Nuclear Medicine VU University Medical Center, Amsterdam, The \\ Netherlands; and ${ }^{5}$ Department of Pathology \& Medical Biology, University of Groningen, University Medical Center Groningen, \\ Groningen, The Netherlands
}

In addition to the well-known estrogen receptor (ER) and human epidermal growth factor receptor 2, the androgen receptor (AR) is also a potential drug target in breast cancer treatment. Whole-body imaging can provide information across lesions within a patient. ER expression in tumor lesions can be visualized by ${ }^{18} \mathrm{~F}$-fluoroestradiol $\left({ }^{18} \mathrm{~F}-\mathrm{FES}\right) \mathrm{PET}$, and AR expression has been visualized in prostate cancer patients with ${ }^{18} \mathrm{~F}$-fluorodihydrotestosterone ( $\left.{ }^{18} \mathrm{~F}-\mathrm{FDHT}\right) \mathrm{PET}$. Our aim was to assess the concordance between ${ }^{18} \mathrm{~F}-\mathrm{FDHT}$ and ${ }^{18} \mathrm{~F}-\mathrm{FES}$ PET and tumor AR and ER expression measured immunohistochemically in patients with metastatic breast cancer. Methods: Patients with ER-positive metastatic breast cancer were eligible for the study, irrespective of tumor AR status. The concordance of ${ }^{18} \mathrm{~F}-\mathrm{FDHT}$ and ${ }^{18} \mathrm{~F}$-FES uptake on PET with immunohistochemical expression of $A R$ and ER in biopsies of corresponding metastases was analyzed. Patients underwent ${ }^{18} \mathrm{~F}$-FDHT PET and ${ }^{18} \mathrm{~F}$-FES PET. A metastasis was biopsied within $8 \mathrm{wk}$ of the PET procedures. Tumor samples with more than $10 \%$ and $1 \%$ nuclear tumor cell staining were considered, respectively, AR- and ER-positive. Correlations between PET uptake and semiquantitative immunohistochemical scoring (percentage positive cells $\times$ intensity) were calculated. The optimum threshold of SUV to discriminate positive and negative lesions for both AR and ER was determined by receiver-operatingcharacteristic analysis. Results: In the 13 evaluable patients, correlation $\left(R^{2}\right)$ between semiquantitative AR expression and ${ }^{18} \mathrm{~F}-\mathrm{FDHT}$ uptake was $0.47(P=0.01)$ and between semiquantitative ER expression and ${ }^{18} \mathrm{~F}$-FES uptake $0.78(P=0.01)$. The optimal cutoff for ARpositive lesions was an $\mathrm{SUV}_{\max }$ of 1.94 for ${ }^{18} \mathrm{~F}-\mathrm{FDHT}$ PET, yielding a sensitivity of $91 \%$ and a specificity of $100 \%$; the optimal cutoff was an SUV $_{\max }$ of 1.54 for ${ }^{18} \mathrm{~F}$-FES PET, resulting in a sensitivity and specificity of $100 \%$ for ER. Conclusion: ${ }^{18} \mathrm{~F}-\mathrm{FDHT}$ and ${ }^{18} \mathrm{~F}-\mathrm{FES}$ uptake correlate well with AR and ER expression levels in representative biopsies. These results show the potential use of whole-body imaging for receptor status assessment, particularly in view of biopsyassociated sampling errors and heterogeneous receptor expression in breast cancer metastases.

Received Mar. 29, 2017; revision accepted Apr. 24, 2017.

For correspondence or reprints contact: Geke A.P. Hospers, Department of Medical Oncology, University Medical Center Groningen, Hanzeplein 1, 9713 GZ Groningen, The Netherlands.

E-mail: g.a.p.hospers@umcg.nl

${ }^{*}$ Contributed equally to this work.

tDeceased.

Published online Sep. 14, 2017.

COPYRIGHT @ 2017 by the Society of Nuclear Medicine and Molecular Imaging.
Key Words: breast cancer; androgen receptor; estrogen receptor; FDHT PET; FES PET

J Nucl Med 2017; 58:1906-1912

DOI: 10.2967/jnumed.117.193649

$\mathbf{T}$ he estrogen receptor (ER) is expressed in $75 \%$ of the breast carcinomas, which makes patients with such tumors eligible for ER-targeted therapy (1). Although the ER, human epidermal growth factor receptor 2, and progesterone receptor are routinely determined in breast cancer for prognosis and treatment decision making, this is not the case for the androgen receptor (AR). Nevertheless, several studies have shown that the AR is also present in $70 \%-80 \%$ of the breast carcinomas, which offers a potential new treatment strategy with AR-affecting drugs (2).

Patients with metastatic breast cancer received androgens in the first half of the 20th century, with response rates of $19 \%-36 \%$ $(3,4)$. However, side effects of androgens, including hirsutism and lowering of voice, combined with awareness of the conversion of androgens into estrogens resulted in the discontinuation of androgen therapy in breast cancer patients. With several emerging, less toxic AR-targeted therapies for patients with prostate cancer, and the development of resistance to current breast cancer treatment options, AR-targeted therapies in breast cancer have reentered clinical trials.

A challenge in this era of rapidly emerging drug targets and treatment options is to administer the right drug to the right patient. It is well recognized that only those patients with ER-expressing tumors can benefit from endocrine therapies (1). Because the ER is functionally and structurally highly comparable to the AR, response to AR-targeting drugs may also rely on AR expression in the tumor.

Standard immunohistochemical staining of the primary tumor is inexpensive, easy to apply, and well established in decision making for adjuvant therapies. However, discordant ER expression between the primary breast tumor and metastases has been observed in $18 \%-40 \%$ of the patients $(5-8)$. Molecular imaging offers the possibility to noninvasively determine the presence of 
relevant drug targets in all metastases within an individual patient. Tumor ER expression can be visualized by ${ }^{18} \mathrm{~F}$-fluoroestradiol $\left({ }^{18} \mathrm{~F}-\mathrm{FES}\right) \mathrm{PET}$ in breast cancer patients (9). AR expression in prostate cancer patients has been evaluated using ${ }^{18} \mathrm{~F}$-fluorodihydrotestosterone $\left({ }^{18} \mathrm{~F}-\mathrm{FDHT}\right)$ PET $(10,11)$. If ${ }^{18} \mathrm{~F}-\mathrm{FDHT}$ PET is also able to determine the AR status in metastatic breast cancer patients, this technique has the potential to select patients eligible for AR-targeted therapies. The aim of the present study was to assess whether uptake on ${ }^{18}$ F-FES and ${ }^{18}$ F-FDHT PET correlates with levels of both ER and AR expression on a simultaneous biopsied metastasis.

\section{MATERIALS AND METHODS}

\section{Patients}

Postmenopausal patients with metastatic breast cancer with previous ER-positive primary tumor were eligible if they had a metastasis outside the liver that was safe to biopsy. Patients were staged with full-body bone scintigraphy (bone scan) and a contrast-enhanced CT scan (chest/abdomen) within $6 \mathrm{wk}$ before the PET examinations. A tumor biopsy was performed within 8 wk of the PET examinations. Exclusion criteria for the study were the use of ER ligands less than 6 wk before entering the study, and a life expectancy of less than 3 mo. Aromatase inhibitors and chemotherapy were allowed. All patients gave written informed consent before study participation, according to the Declaration of Helsinki, and the protocol was approved by the local Ethical Committee (EudraCT no., 2012-003981-42)

\section{Study Design}

We performed a prospective, 2-center feasibility trial (NCT01988324). The primary endpoint was the concordance of ${ }^{18} \mathrm{~F}$-FDHT and ${ }^{18} \mathrm{~F}-\mathrm{FES}$ uptake with, respectively, AR and ER expression in a newly obtained biopsy of a metastasis measured by immunohistochemistry. Secondary endpoints were the optimum threshold to discriminate positive and negative lesions for both AR and ER on PET, inter- and intrapatient ${ }^{18} \mathrm{~F}$-FDHT and ${ }^{18} \mathrm{~F}$-FES heterogeneity, and correlation between tracer uptake and serum hormone levels at the time of scanning. Venous blood was collected before ${ }^{18} \mathrm{~F}$-FES tracer injection to evaluate serum estradiol (luminescence immune assay), luteinizing hormone, folliclestimulating hormone (both fluorescence immune assay), and sex hormone-binding globulin (chemiluminescence microparticle immune assay). These have been reported to affect tumor ${ }^{18} \mathrm{~F}$-FES uptake in breast cancer studies (12). Before ${ }^{18} \mathrm{~F}$-FDHT injection, blood was collected for serum testosterone and dihydrotestosterone levels (both liquid chromatography-mass spectrometry assay).

\section{Tumor Histology}

All patients underwent a biopsy of a metastasis, detectable by conventional imaging, within 8 wk of the PET procedures. Biopsies were formalin-fixed and paraffin-embedded. Biopsies were centrally revised by a dedicated breast pathologist. ER (CONFIRM antiEstrogen Receptor [SP1] Rabbit Monoclonal Primary Antibody; Ventana) and AR (anti-Androgen Receptor [SP107] Rabbit Monoclonal Primary Antibody; Ventana) were stained with a Benchmark automated stainer (Ventana) at the Department of Pathology of the University Medical Center Groningen. Antibodies were prediluted by the supplier. ER was scored according to the guideline of the American Society of Clinical Oncologypathologists (13) and semiquantitatively: the percentage of positive tumor nuclei was multiplied by the intensity of staining ( 0 , negative; 1 , weak; 2 , moderate; and 3 , strong). This led to a score of $0-300$ (14). Because AR is not a routine staining in breast cancer, a threshold of more than $10 \%$ positive nuclear staining was used as a discriminator for AR positivity, based on current use in literature (2).
TABLE 1

Patient Characteristics

\begin{tabular}{|c|c|c|}
\hline Characteristic & $n$ & $\%$ \\
\hline Mean age $(y)$ & 64 & \\
\hline \multicolumn{3}{|l|}{ Sex } \\
\hline Female & 11 & 85 \\
\hline Male & 2 & 15 \\
\hline \multicolumn{3}{|c|}{ Primary tumor characteristics IHC } \\
\hline $\mathrm{ER}+/ \mathrm{AR}+$ & 13 & 100 \\
\hline $\mathrm{ER}+/ \mathrm{AR}-$ & 0 & \\
\hline $\mathrm{ER}-/ \mathrm{AR}+$ & 0 & \\
\hline ER-/AR- & 0 & \\
\hline \multicolumn{3}{|l|}{ Primary tumor stage } \\
\hline T1N0M0 & 4 & 31 \\
\hline T1N1M0 & 1 & 8 \\
\hline T2NOMO & 4 & 31 \\
\hline T2N1M0 & 1 & 8 \\
\hline T3N2MO & 3 & 23 \\
\hline \multicolumn{3}{|c|}{ Metastatic tumor characteristics IHC } \\
\hline $\mathrm{ER}+/ \mathrm{AR}+$ & 10 & 77 \\
\hline $\mathrm{ER}+/ \mathrm{AR}-$ & 1 & 8 \\
\hline ER-/AR+ & 1 & 8 \\
\hline ER-/AR- & 1 & 8 \\
\hline \multicolumn{3}{|c|}{$\begin{array}{l}\text { Treatment at time of }{ }^{18} \mathrm{~F}-\mathrm{FES} \text { and } \\
{ }^{18} \mathrm{~F}-\mathrm{FDHT} \text { PET scans }\end{array}$} \\
\hline Aromatase inhibitor & 5 & 38 \\
\hline Chemotherapy & 4 & 31 \\
\hline None & 4 & 31 \\
\hline
\end{tabular}

$\mathrm{ER}+$ = ER-positive; AR $+=$ AR-positive; ER- = ER-negative; AR- = AR-negative; IHC = immunohistochemistry.

\section{Imaging}

CT scans were evaluated by a radiologist. Bone scans were evaluated by 2 nuclear physicians. All tumor lesions visible on CT $(>1 \mathrm{~cm})$ and bone scans were documented. Patients underwent ${ }^{18} \mathrm{~F}$-FDHT PET and ${ }^{18} \mathrm{~F}$-FES PET on separate days within $14 \mathrm{~d}$. ${ }^{18} \mathrm{~F}$-FES and ${ }^{18} \mathrm{~F}$-FDHT were produced as described previously $(15,16)$. Patients received approximately $200 \mathrm{MBq}$ of ${ }^{18} \mathrm{~F}$-FDHT and ${ }^{18}$ F-FES. Whole-body PET/CT was performed $60 \mathrm{~min}$ after tracer injection with a Siemens 64-slice mCT (PET/CT) (University Medical Center Groningen) or a Philips Gemini 64 TF PET/CT camera (VU University Medical Center) using the European Association of Nuclear Medicine Research Limited (EARL)-approved protocols (17). Low-dose CT (for attenuation and scatter correction) and PET imaging were performed within 1 procedure. All images were reconstructed according to the specifications of the EARL accreditation program (17).

Tumor ${ }^{18} \mathrm{~F}$-FES uptake was quantified for all lesions seen on CT and bone scans, as well as for nonphysiologic uptake visible above background with an $\mathrm{SUV}_{\max }$ of greater than 1.5 based on previous studies $(18,19)$. All lesions detected on bone, CT, and ${ }^{18} \mathrm{~F}$-FES PET scans were also quantified on the ${ }^{18} \mathrm{~F}$-FDHT PET scan. In line with previous studies, we used the $\mathrm{SUV}_{\text {max }}$ to calculate tumor ${ }^{18} \mathrm{~F}-\mathrm{FDHT}$ and ${ }^{18}$ F-FES uptake $(18,19)$. We also measured the $\operatorname{SUV}_{\text {mean }}$ using a 

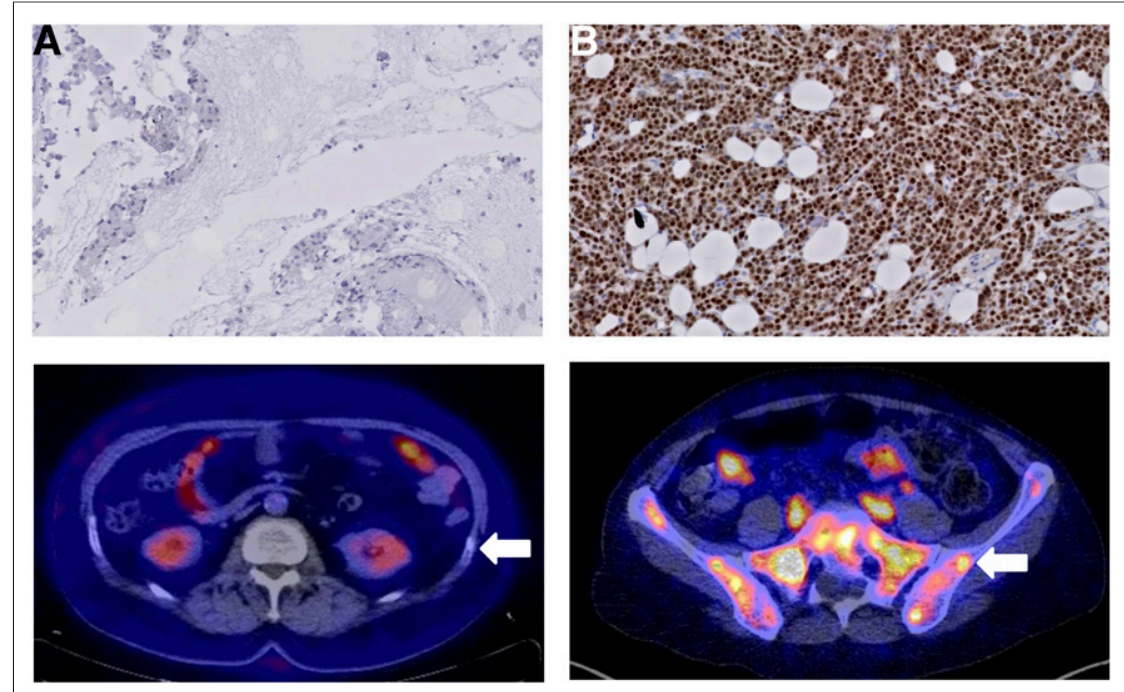

respectively. The optimum threshold of SUV to discriminate positive and negative lesions for both AR and ER was determined by receiver-operating-characteristic analysis. Correlations between semiquantitative receptor analysis and SUV were calculated using the Pearson correlation coefficient. A $P$ value of 0.05 or less was considered significant.

\section{RESULTS}

\section{Patient Characteristics}

Twenty-one patients were included between September 2014 and August 2015 (Table 1), and 13 were evaluable for the primary study endpoint. Nonevaluable were 5 patients with a nonvital tumor biopsy (24\%), and 3 patients (14\%) with biopsied lesions not visible on conventional imaging or PET ( $n=2$ skin lesions, $n=1$ intestinal lesion). All evaluable patients had an ER-positive and AR-positive primary breast cancer based on immunohistochemistry. Three patients (23\%) showed conversion between the primary tumor and the metastasis of

$50 \%$ isocontour of the hottest pixel to assess the average SUV computed in the volume of interest. The SUV $\mathrm{Seak}_{\text {pas }}$ used to calculate uptake in a $1-\mathrm{cm}^{3}$ spheric volume of interest surrounding the voxel with the highest activity. Background correction was applied using a volume of interest at the unaffected contralateral site whenever available, or at the surrounding tissue of the same origin and deducted from the SUV of the tumor (i.e., lesion $\mathrm{SUV}_{\text {max/mean/peak }}$ minus background $\mathrm{SUV}_{\max / \text { mean/peak }}$, resulting in background corrected $\mathrm{SUV}_{\text {max/mean/peak }}$.

\section{Statistical Analysis}

${ }^{18} \mathrm{~F}$-FDHT PET/CT and ${ }^{18} \mathrm{~F}$-FES PET/CT findings were compared with immunohistochemical findings for $\mathrm{AR}$ and ER expression, either ER (8\%), AR (8\%), or both $(8 \%)$ measured with immunohistochemistry.

\section{Concordance Between SUV and Immunohistochemistry of Same Tumor Lesion}

Figure 1 shows 2 representative examples of AR immunohistochemical staining results and corresponding ${ }^{18} \mathrm{~F}-\mathrm{FDHT}$ PET scans. Mean ${ }^{18} \mathrm{~F}$-FDHT $\mathrm{SUV}_{\max }$ of the biopsies in AR-positive lesions was 3.1 ( $\mathrm{SD}, 0.90)$ versus a mean ${ }^{18} \mathrm{~F}-\mathrm{FDHT} \mathrm{SUV}_{\max }$ in ARnegative lesions of 1.9 (SD, 0.01). Mean ${ }^{18} \mathrm{~F}-\mathrm{FES} \mathrm{SUV}_{\max }$ of the biopsied ER-positive lesions was 4.3 (SD, 2.4) versus a mean
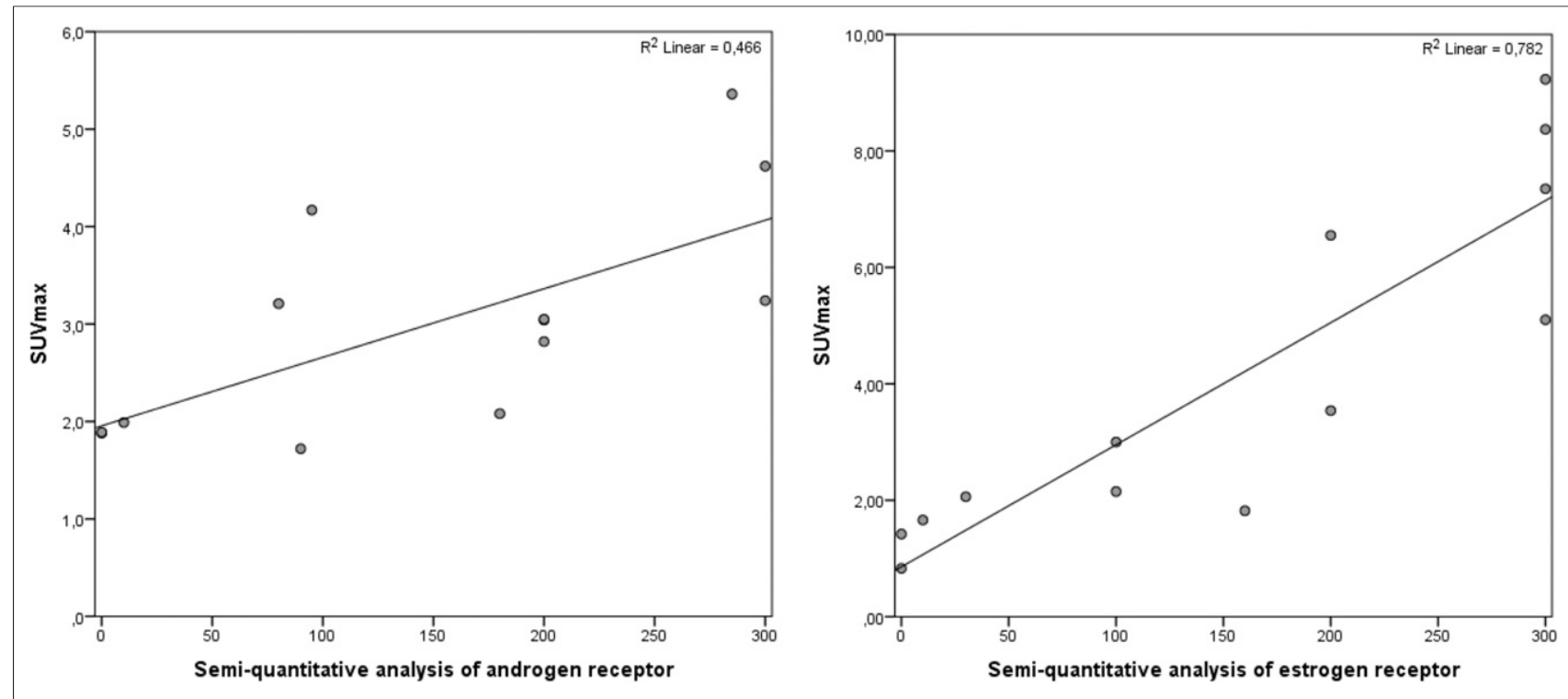

FIGURE 2. Correlation plot of semiquantitative analysis of receptor status and SUV max as measured by PET scan for AR (left) and ER (right). 


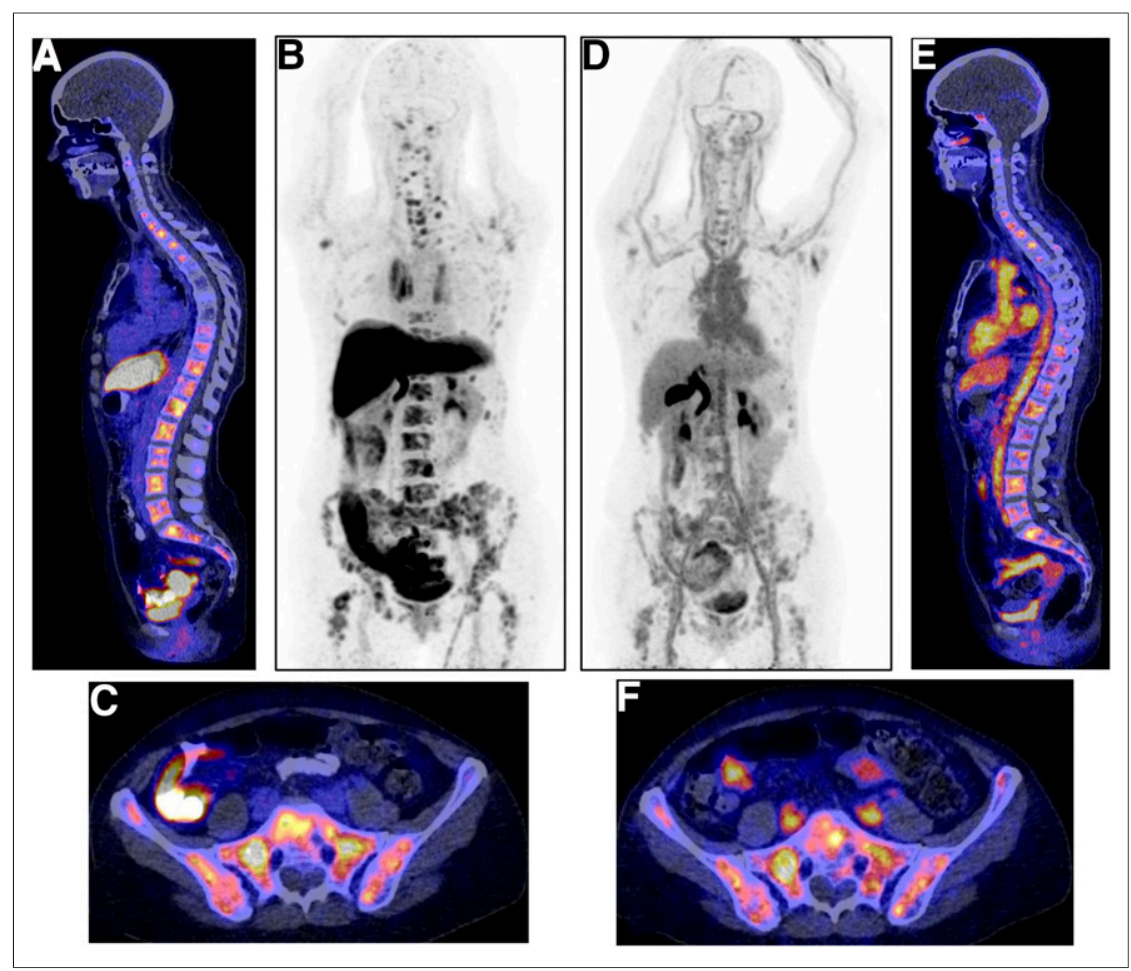

FIGURE 3. Example of typical ${ }^{18} \mathrm{~F}-\mathrm{FES}(\mathrm{A}-\mathrm{C})$ and ${ }^{18} \mathrm{~F}-\mathrm{FDHT}(\mathrm{D}-\mathrm{F})$ distribution in same patient with multiple bone metastases. (A) Sagittal ${ }^{18} \mathrm{~F}$-FES PET/CT fusion image with physiologic uptake in liver, small intestine, and urinary tract and pathologic uptake in multiple vertebra. (B) ${ }^{18} \mathrm{~F}$-FES PET maximum-intensity-pixel format to allow visualization of biodistribution of ${ }^{18}$ F-FES tracer. (C) Horizontal ${ }^{18} \mathrm{~F}$-FES PET/CT fusion image with physiologic uptake in small intestine and pathologic uptake throughout pelvic bones. (D) Maximum-intensity-pixel format of ${ }^{18} \mathrm{~F}-\mathrm{FDHT}$ PET scan, with physiologic uptake in blood pool of heart and liver and excretion via bile to small intestine, and urinary tract. (E) Sagittal ${ }^{18} \mathrm{~F}$-FDHT PET/CT fusion image with physiologic uptake and pathologic uptake in multiple vertebrae. (F) Horizontal ${ }^{18} \mathrm{~F}$-FES PET/CT fusion image with physiologic uptake in large vessels and small intestines and pathologic uptake throughout pelvic bones.

${ }^{18} \mathrm{~F}_{-\mathrm{FES} \mathrm{SUV}}$ max in biopsied ER-negative lesions of 1.1 (SD, 0.4). The correlation between semiquantitative AR expression and ${ }^{18} \mathrm{~F}-\mathrm{FDHT}$ uptake was $R^{2}=0.47(P=0.01)$, and between semiquantitative ER expression and ${ }^{18} \mathrm{~F}-\mathrm{FES}$ uptake it was $R^{2}=0.78$ $(P=0.01)$ (Fig. 2). The correction for background uptake did not improve the correlation between semiquantitative AR and ER expression and ${ }^{18} \mathrm{~F}$-FDHT and ${ }^{18} \mathrm{~F}$-FES uptake, because background correction resulted in a correlation of $R^{2}=0.39$ and 0.78 , respectively. The correlations between immunohistochemistry and $\mathrm{SUV}_{\text {peak }}, \mathrm{SUV}_{\text {mean }}$, and background-corrected $\mathrm{SUV}_{\text {peak }}$ and $\mathrm{SUV}_{\text {mean }}$ did not differ from the correlations observed between immunohistochemistry and SUV max $_{\text {(Supple- }}$ mental Table 1; supplemental materials are available at http:// jnm.snmjournals.org).

The optimal $\mathrm{SUV}_{\max }$ cutoff for ${ }^{18} \mathrm{~F}-\mathrm{FDHT}$ PET was 1.9 , leading to a sensitivity of $91 \%$ and a specificity of $100 \%$ (area under the curve, $0.91 ; 95 \%$ confidence interval, 0.74-1.0). Receiver-operating-characteristic analysis showed an optimal cutoff value for ${ }^{18} \mathrm{~F}$-FES PET to be $\mathrm{SUV}_{\max } 1.5$, resulting in a sensitivity and specificity of $100 \%$. The correction of tracer uptake for background or using $S_{U V}$ mean or $S U V_{\text {peak }}$ instead of $\mathrm{SUV}_{\max }$ did not improve the results (Supplemental Table 2).

\section{Heterogeneity in Uptake}

Heterogeneity in lesion uptake was seen between patients and across lesions within individual patients for both ${ }^{18} \mathrm{~F}-\mathrm{FES}$ and ${ }^{18} \mathrm{~F}$-FDHT uptake. An example of a typical ${ }^{18} \mathrm{~F}$-FDHT and ${ }^{18} \mathrm{~F}$-FES PET is shown in Figure 3. With the cutoff at 1.9 for ${ }^{18} \mathrm{~F}$-FDHT PET, all patients had both ${ }^{18} \mathrm{~F}$-FDHT-positive and ${ }^{18} \mathrm{~F}$-FDHTnegative lesions. SUV $\max$ on ${ }^{18}$ F-FDHT for tumor lesions varied within patients (median, 2.8; range, 0.8-6.5) and between patients (median, 2.7; range, 1.7-3.7). Eleven of 13 patients had visually both ${ }^{18} \mathrm{~F}$-FES-positive and ${ }^{18} \mathrm{~F}-\mathrm{FES}-$ negative lesions. SUV max $_{\text {max }}$ on ${ }^{18} \mathrm{~F}$-FES PET varied widely between lesions (median, 3.2; range, 0.6-12.2) and patients (median, 2.4; range, 1.3-6.0).

A total of 298 lesions were detected with CT scans $(n=95)$, bone scans $(n=126)$, or ${ }^{18}$ F-FES PET scans $(n=239)$. Most lesions $(81 \%)$ showed uptake above background on ${ }^{18}$ F-FES PET. CT or bone scan identified 59 lesions that showed no ${ }^{18} \mathrm{~F}-\mathrm{FES}$ uptake above background. ${ }^{18}$ F-FES PET identified 48 lesions not visible on conventional imaging. In total, 278 lesions could be used for ${ }^{18}$ F-FES PET analysis. Because of the high physiologic background uptake near the lesion such as in the liver and intestines, 20 lesions could not be reliably quantified. Most of the lesions were in the bone $(n=219) ; 34$ lesions were in the lymph nodes, and 25 were visceral lesions (Fig. 4).

On ${ }^{18}$ F-FDHT PET scans, 196 lesions (66\%) were visible above background, of which 42 lesions could not be reliably quantified because of high physiologic background uptake near the lesion (e.g., the liver, blood vessels, and intestines). One hundred two lesions were not visible above background but were visible on either CT scan, bone scan, or ${ }^{18}$ F-FES PET scan. In total, 256 lesions were included for ${ }^{18} \mathrm{~F}$-FDHT PET analysis. Most of the lesions were bone lesions $(n=222), 14$ lesions were lymph nodes, and the remaining 20 lesions were visceral lesions (Fig. 5).

Uptake in healthy liver tissue was high on both ${ }^{18} \mathrm{~F}$-FDHT PET and ${ }^{18}$ F-FES PET scans, rendering analysis of liver metastases impossible. The mean liver uptake on ${ }^{18} \mathrm{~F}$-FDHT $\mathrm{SUV}_{\text {mean }}$ was 4.4 (range, 3.6-5.8) versus the mean liver uptake on ${ }^{18}$ F-FES SUV mean of 12.8 (range, 8.2-19.6). Several lesions were nonquantfiable due to high blood-pool accumulation on the ${ }^{18}$ F-FDHT PET. The blood-pool accumulation measured in the descending thoracic artery was higher on ${ }^{18} \mathrm{~F}$-FDHT PET than ${ }^{18} \mathrm{~F}$-FES PET: SUV mean of 4.6 (range, 3.8-6.2) versus $\mathrm{SUV}_{\text {mean }}$ of 1.3 (range, 0.9-2.1), respectively.

\section{Correlation Between PET Uptake and Serum Hormone Levels and Sex Hormone-Binding Globulin}

${ }^{18} \mathrm{~F}$-FDHT tumor uptake did not correlate with serum sex hormone-binding globulin, DHT, or testosterone levels 


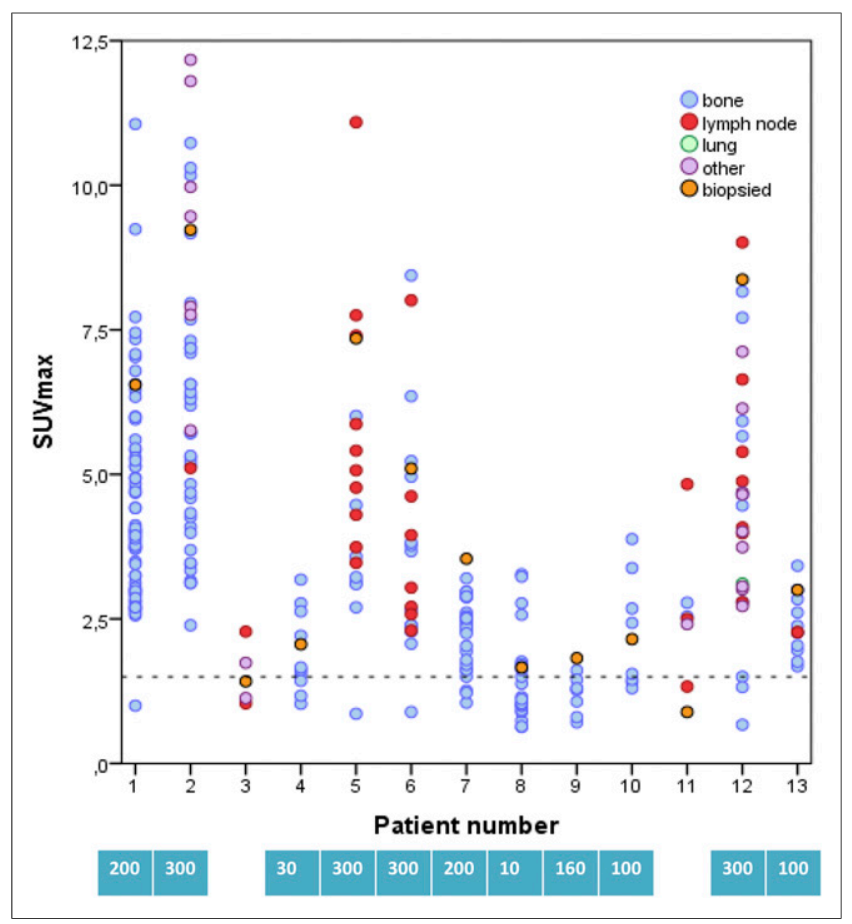

FIGURE 4. Distribution of SUV $\mathrm{Vmax}_{\text {max }}$ per lesion per patient measured by ${ }^{18} \mathrm{~F}-\mathrm{FES}$ PET. Lesions are divided into bone (blue), lymph nodes (red), lung (green), and others (purple). Orange circles are biopsied lesions. Blue boxes indicate ER-positive biopsies ( $>1 \%$ staining); numbers indicate score of biopsy (i.e., intensity times percentage positive cells). Dashed line indicates threshold set based on receiver-operatingcharacteristic analysis. White boxes indicate negative biopsies $(<1 \%$ staining).

(Supplemental Tables 3-5). Serum estradiol levels correlated positively with ${ }^{18} \mathrm{~F}$-FES tumor uptake $\left(R^{2}=0.52 ; P=0.01\right) .{ }^{18} \mathrm{~F}-\mathrm{FES}$ tumor uptake did not correlate with sex hormone-binding globulin, luteinizing hormone, or follicle-stimulating hormone serum levels.

\section{DISCUSSION}

This is the first study in which the ${ }^{18} \mathrm{~F}-\mathrm{FDHT}$ uptake is evaluated in breast cancer patents and in which ${ }^{18} \mathrm{~F}$-FDHT tracer uptake was correlated with semiquantitative AR analysis in a biopsy of the corresponding metastasis. ${ }^{18} \mathrm{~F}$-FDHT uptake shows a moderate correlation with AR expression, and ${ }^{18} \mathrm{~F}$-FES uptake shows a strong correlation with ER expression.

In this study, we showed that ${ }^{18} \mathrm{~F}-\mathrm{FDHT}$ can identify AR-positive metastases in breast cancer patients. ${ }^{18} \mathrm{~F}$-FDHT PET may therefore be an interesting tool to select patients eligible for clinical trials with AR antagonists and to analyze the receptor occupancy of these drugs. AR-targeted therapy is not yet standard in breast cancer patients, but preliminary results of phase II trials are promising, with stable disease in $35 \%$ of metastatic breast cancer patients $(20,21)$. More clinical studies exploring the efficacy of AR-targeted therapy in AR-positive metastatic breast cancer are currently ongoing (e.g., NCT00468715, NCT00755885). Even combined AR- and ER-targeted therapies are currently under way (NCT02910050, NCT02953860).

To date ${ }^{18}$ F-FDHT PET has been used only in trials with metastatic prostate cancer patients. A comparison of 59

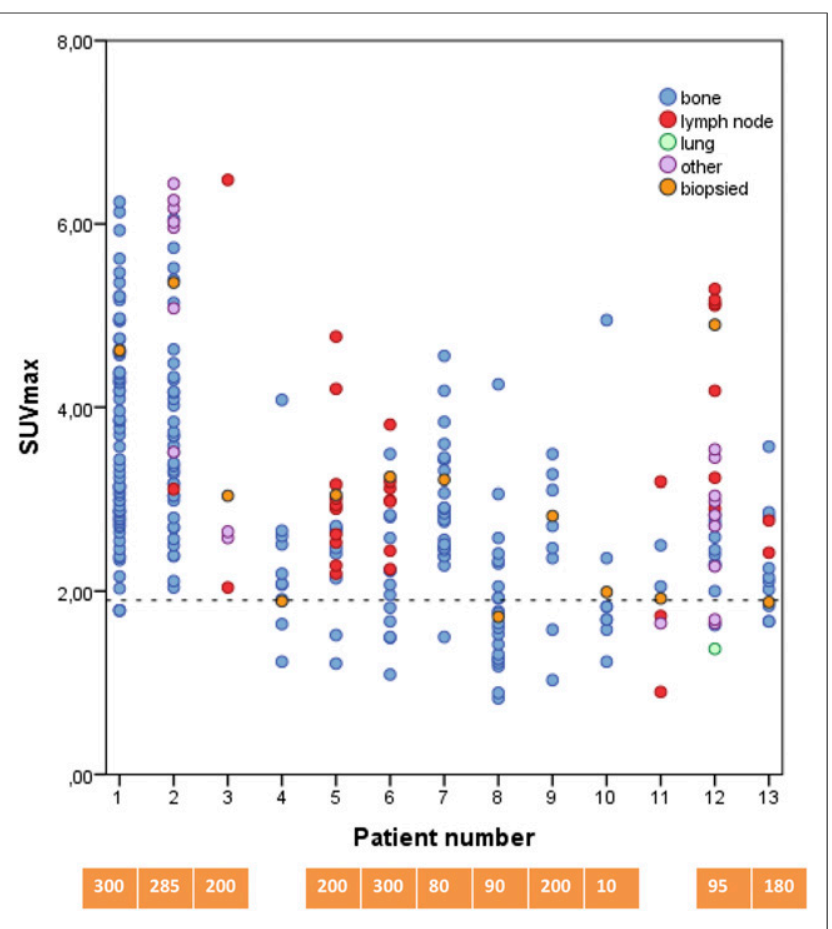

FIGURE 5. Distribution of $\mathrm{SUV}_{\max }$ per lesion per patient measured by ${ }^{18} \mathrm{~F}-\mathrm{FDHT}$ PET. Lesions are divided into bone (blue), lymph nodes (red), lung (green), and others (purple). Orange circles are biopsied lesions. Orange boxes indicate AR-positive biopsies (i.e., $>10 \%$ staining); white boxes indicate negative biopsies. Numbers in boxes indicate score of biopsy (i.e., intensity times percentage positive cells). Dashed line indicates threshold set based on receiver-operating-characteristic analysis.

metastatic prostate cancer lesions visible on conventional imaging showed that $97 \%$ were also visible on the ${ }^{18} \mathrm{~F}$-FDHT PET (10). Here, conventional imaging also included ${ }^{18}$ F-FDG PET. In our study, we found that $66 \%$ of the lesions visible on conventional imaging were visible on ${ }^{18} \mathrm{~F}$-FDHT PET.

With serial ${ }^{18}$ F-FES PET scans in patients treated with ER modulators such as fulvestrant, we were able to visualize residual ER availability during therapy, which was associated with early progression (22). For other ER modulators such as GDC0810 and Z-endoxifen, ${ }^{18} \mathrm{~F}$-FES PET provided information about ER occupancy and guided dose selection for phase II trials $(23,24) .{ }^{18}$ F-FDHT uptake in tumor lesions of patients with prostate cancer diminished in 3 patients after treatment with a high dose of testosterone. Treatment with the AR blocker enzalutamide also resulted in a reduced uptake on ${ }^{18} \mathrm{~F}$-FDHT PET in prostate cancer patients (10). We are currently investigating the effect of the AR blocker bicalutamide on residual AR availability assessed by ${ }^{18} \mathrm{~F}$-FDHT PET in patients with metastatic breast cancer. Secondary endpoints are the relation between percentage decreased uptake on ${ }^{18} \mathrm{~F}$-FDHT PET and response to treatment measured by RECIST in the case of measurable disease (NCT02697032).

This study enforces the earlier observed correlation between ${ }^{18} \mathrm{~F}$-FES uptake and ER expression. Correlations between the ${ }^{18}$ F-FES PET uptake parameters and immunohistochemistry on the metastatic biopsy using an $\mathrm{SUV}_{\max }$ of greater than 1.5 showed a $100 \%$ sensitivity and specificity similar to previously published results $(9,18,19)$. The parameters $\mathrm{SUV}_{\text {max }}, \mathrm{SUV}_{\text {mean }}$, 
and $\mathrm{SUV}_{\text {peak }}$ did not differ, and correction for background did not influence the correlation. Therefore, for ${ }^{18} \mathrm{~F}$-FES PET analysis in the diagnostic setting $\mathrm{SUV}_{\max }$ can be used, and correction for background is not required. The correlation between uptake on ${ }^{18}$ F-FDHT PET scans and immunohistochemical staining was lower than on ${ }^{18} \mathrm{~F}$-FES PET and immunohistochemical staining. The kinetic properties and metabolism of ${ }^{18} \mathrm{~F}$-FES and ${ }^{18} \mathrm{~F}$-FDHT are similar $(25,26)$. But ${ }^{18} \mathrm{~F}$-FDHT has a lower relative binding affinity of 0.43 for AR than FES, which has a binding affinity of 0.83 for ER (21). Furthermore, SUV might not be the best quantification method for ${ }^{18} \mathrm{~F}$-FDHT uptake. In a small study with 4 metastatic prostate cancer patients, SUV corrected for plasma ${ }^{18} \mathrm{~F}$-FDHT concentration showed a better correlation (27).

We analyzed factors that potentially could influence tracer uptake such as circulating hormone levels. We found only estradiol levels to be correlated with higher uptake on ${ }^{18} \mathrm{~F}$-FES PET scans, which might be related to higher ER expressions in tumor lesions in postmenopausal patients with higher residual estradiol levels. In fact, we found a correlation of $R^{2}$ of 0.42 $(P=0.02)$ between serum estradiol levels and ER expression determined by immunohistochemical staining on a metastasis biopsy. There was no correlation between other serum hormone levels and ${ }^{18} \mathrm{~F}$-FES or ${ }^{18} \mathrm{~F}$-FDHT tumor uptake. These data indicate that physiologic circulating hormone levels are too low to directly affect tracer uptake in the tumor. Tracer uptake can be influenced by volume, that is, partial-volume effect, where smaller tumor sizes results in an underestimation of uptake (28). However, in our study there was no correlation found between the volumes of interest of the lesions and ${ }^{18} \mathrm{~F}-\mathrm{FES}$ or ${ }^{18}$ F-FDHT uptake.

Because of the feasibility setting of this study, only a limited number of patients were evaluable for primary endpoint. Therefore, larger studies should confirm the optimal cutoff value for ${ }^{18}$ F-FDHT PET. In our study, 5 of the 21 entered patients did not have vital tumor tissue in their metastatic biopsies. CT may have also shown bone lesions that were no longer active, as patients were heavily pretreated. This might have resulted in an overestimation of ${ }^{18} \mathrm{~F}$-FES- and ${ }^{18} \mathrm{~F}$-FDHT-negative sites. Others have used ${ }^{18} \mathrm{~F}$-FDG PET to visualize hormone receptor-negative lesions. We refrained from doing so, because ${ }^{18} \mathrm{~F}-\mathrm{FDG}$ PET can also be negative in hormone receptor-positive breast cancer lesions (29). PET imaging of hormone receptors also has some restrictions. Liver lesions are nonevaluable by ${ }^{18} \mathrm{~F}-\mathrm{FES}$ and ${ }^{18} \mathrm{~F}-\mathrm{FDHT}$ PET because of high uptake of both tracers in the liver. In addition, ${ }^{18} \mathrm{~F}$-FDHT PET has the disadvantage of high accumulation in the blood pool, rendering it difficult to analyze lesions near large veins. This has also been described in a ${ }^{18} \mathrm{~F}$-FDHT PET study in prostate cancer patients (9). If the ${ }^{18} \mathrm{~F}$-FDHT PET would be used as a diagnostic tool, this would be complementary to the current conventional imaging.

\section{CONCLUSION}

In our heavily pretreated patient population, hormone receptor conversion in the metastasis, when compared with the primary tumor, occurred in $23 \%$ of the patients. This is similar to previously reported conversion rates $(7,8)$. Heterogeneous uptake in tumor lesions on both ${ }^{18} \mathrm{~F}$-FES and ${ }^{18} \mathrm{~F}$-FDHT PET was seen in most patients, suggesting that both receptor-positive and -negative lesions are present in 1 patient. Current guidelines advise on a biopsy being performed when metastatic disease presents. This may not always be feasible. However, when omitted, changes in receptor status over time might lead to suboptimal therapy choices. ${ }^{18} \mathrm{~F}-\mathrm{FDHT}$ and ${ }^{18} \mathrm{~F}$-FES PET have the potential to serve as a surrogate for metastasis biopsy, especially when lesions are difficult to access or sampling errors are prone to occur.

\section{DISCLOSURE}

This work was supported by CTMM-MAMMOTH WP5, the Alpe d'HuZes/Dutch Cancer Society (RUG 2013-5960), the Ubbo Emmius Fund grant (510215), the van der Meer-Boerema Foundation, and the Anna Dorothea Hingst Foundation. No other potential conflict of interest relevant to this article was reported.

\section{REFERENCES}

1. Blamey RW, Hornmark-Stenstam B, Ball G, et al. ONCOPOOL: a European database for 16,944 cases of breast cancer. Eur J Cancer. 2010;46:56-71.

2. Collins LC, Cole KS, Marotti JD, Hu R, Schnitt SJ, Tamimi RM. Androgen receptor expression in breast cancer in relation to molecular phenotype: results from the Nurses' Health Study. Mod Pathol. 2011;24:924-931.

3. Adair FE, Herrmann JB. The use of testosterone propionate in the treatment of advanced carcinoma of the breast. Ann Surg. 1946;123:1023-1035.

4. Olson KB. Fluoxymesterone (halotestin) in the treatment of advanced breast cancer. N Y State J Med. 1959;59:248-252.

5. Layfield LJ, Saria E, Mooney EE, Liu K, Dodge RR. Tissue heterogeneity of immunohistochemically detected estrogen receptor: implications for image analysis quantification. Am J Clin Pathol. 1998;110:758-764.

6. Chung GG, Zerkowski MP, Ghosh S, Camp RL, Rimm DL. Quantitative analysis of estrogen receptor heterogeneity in breast cancer. Lab Invest. 2007;87:662-669.

7. Simmons C, Miller N, Geddie W, et al. Does confirmatory tumor biopsy alter the management of breast cancer patients with distant metastases. Ann Oncol. 2009;20:1499-1504.

8. Amir E, Miller N, Geddie W, et al. Prospective study evaluating the impact of tissue confirmation of metastatic disease in patients with breast cancer. $J$ Clin Oncol. 2012;30:587-592.

9. Linden HM, Stekhova SA, Link JM, et al. Quantitative fluoroestradiol positron emission tomography imaging predicts response to endocrine treatment in breast cancer. J Clin Oncol. 2006;24:2793-2799.

10. Larson SM, Morris M, Gunther I, et al. Tumor localization of $16 \beta^{-18}$ F-fluoro- $5 \alpha-$ dihydrotestosterone versus ${ }^{18} \mathrm{~F}-\mathrm{FDG}$ in patients with progressive, metastatic prostate cancer. J Nucl Med. 2004;45:366-373.

11. Scher HI, Beer TM, Higano CS, et al. Antitumour activity of MDV3100 in castration-resistant prostate cancer: a phase 1-2 study. Lancet. 2010;375:1437-1446.

12. Peterson LM, Kurland BF, Link JM, et al. Factors influencing the uptake of ${ }^{18} \mathrm{~F}$-fluoroestradiol in patients with estrogen receptor positive breast cancer. Nucl Med Biol. 2011;38:969-978.

13. Hammond MEH, Hayes DF, Dowsett M, et al. American Society of Clinical Oncology/College of American Pathologists guideline recommendations for immunohistochemical testing of estrogen and progesterone receptor in breast cancer. J Clin Oncol. 2010;28:2784-2795.

14. McCarty KS Jr, Miller LS, Cox EB, Konrath J, McCarty KS Sr. Estrogen receptor analyses. Correlation of biochemical and immunohistochemical methods using monoclonal antireceptor antibodies. Arch Pathol Lab Med. 1985;109:716-721.

15. Liu A, Dence CS, Welch MJ, Katzenellenbogen JA. Fluorine-18-labeled androgens: radiochemical synthesis and tissue distribution studies on six fluorinesubstituted androgens, potential imaging agent for prostatic cancer. $\mathrm{J} \mathrm{Nucl}$ Med. 1992;33:724-734.

16. Römer J, Steinbach J, Kasch H. Studies on the synthesis of $16 \alpha\left[{ }^{18} \mathrm{~F}\right]$ fluoroestradiol. Appl Radiat Isot. 1996;47:395-399.

17. Boellaard R, Delgado-Bolton R, Oyen WJ, et al. FDG PET/CT: EANM procedure guidelines for tumour imaging: version 2.0. Eur J Nucl Med Mol Imaging. 2015;42:328-354.

18. Venema CM, Apollonio G, Hospers GA, et al. Recommendations and technical aspects of $16 \alpha-\left[{ }^{18} \mathrm{~F}\right]$ fluoro-17 $\beta$ estradiol PET to image the estrogen receptor in vivo: the Groningen experience. Clin Nucl Med. 2016;41:844-851.

19. van Kruchten M, de Vries EG, Brown M, et al. PET imaging of oestrogen receptors in patients with breast cancer. Lancet Oncol. 2013;14:e465-e475.

20. Overmoyer B, Sanz-Altamira P, Taylor RP, et al. Enobosarm: a targeted therapy for metastatic, androgen receptor positive, breast cancer [abstract]. J Clin Oncol. $2014 ; 32: 5 \mathrm{~S}$. 
21. Traina TA, O'Shaughnessy J, Kelly CM, et al. A phase 2 single-arm study of the clinical activity and safety of enzalutamide in patinets with advanced androgen-receptor-positive triple-negative breast cancer [abstract]. J Clin Oncol. 2014;32:5S.

22. van Kruchten M, de Vries EG, Glaudemans AW, et al. Measuring residual estrogen receptor availability during fulvestrant therapy in patients with metastatic breast cancer. Cancer Discov. 2015;5:72-81.

23. Lin FI, Gonzales EM, Kummar S, et al. Utility of ${ }^{18} \mathrm{~F}$-fluoroestradiol ( ${ }^{18} \mathrm{~F}-\mathrm{FES}$ ) $\mathrm{PET} / \mathrm{CT}$ imaging as a pharmacodynamic marker in patients with refractory estrogen receptor-positive solid tumors receiving Z-endoxifen therapy. Eur J Nucl Med Mol Imaging. 2017;44:500-508.

24. Wang Y, Ayres K, Goldman DA, et al. ${ }^{18}$ F-fluoroestradiol PET/CT measurement of estrogen receptor suppression during a phase I trial of the novel estrogen receptor targeted therapeutic GDC-0810. Clin Cancer Res. 2017;23: 3053-3060.
25. Beattie BJ, Smith-Jones PM, Jhanwar YS, et al. Pharmacokinetic assessment of the uptake of $16 \alpha-{ }^{18} \mathrm{~F}$-fluoro-5 $\alpha$-dihidrotestosterone in prostate tumors as measured by PET. J Nucl Med. 2010;51:183-192.

26. Kumar P, Mercer J, Doerkson C, Tonkin K, McEwan AJ. clinical production, stability studies and PET imaging with $16 \alpha-\left[{ }^{18} \mathrm{~F}\right]$ fluoroestradiol $\left(\left[{ }^{18} \mathrm{~F}\right] \mathrm{FES}\right)$ in ER positive breast cancer patients. J Pharm Pharm Sci. 2007;10:256s.

27. Kramer G, Yaqub M, Schuit R, et al. Assessment of simplified methods for quantification of ${ }^{18} \mathrm{~F}$-FDHT uptake in patients with metastasized castrate resistant prostate cancer [abstract]. J Nucl Med. 2016;57(suppl 2):464.

28. Soret M, Bacharach SL, Buvat I. Partial volume effect in PET tumor imaging. J Nucl Med. 2007;48:932-945.

29. Groheux D, Majdoub M, Tixier F, et al. Do clinical, histological or immunohistochemical primary tumour characteristics translate into different ${ }^{18} \mathrm{~F}$-FDG $\mathrm{PET} / \mathrm{CT}$ volumetric and heterogeneity features in stage II/III breast cancer? Eur J Nucl Med Mol Imaging. 2015;42:1682-1691. 